Supplement of Atmos. Chem. Phys., 20, 8381-8404, 2020

https://doi.org/10.5194/acp-20-8381-2020-supplement

(c) Author(s) 2020. This work is distributed under

the Creative Commons Attribution 4.0 License.

(c) (i)

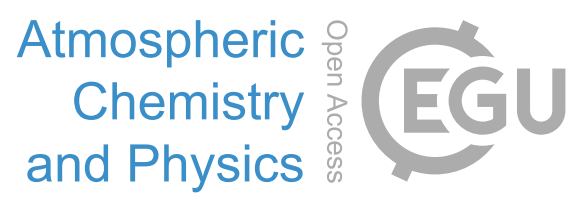

Supplement of

\title{
Fast responses on pre-industrial climate from present-day aerosols in a CMIP6 multi-model study
}

Prodromos Zanis et al.

Correspondence to: Prodromos Zanis (zanis@geo.auth.gr)

The copyright of individual parts of the supplement might differ from the CC BY 4.0 License. 
ERF DJF [piClim_aer - piClim_control]
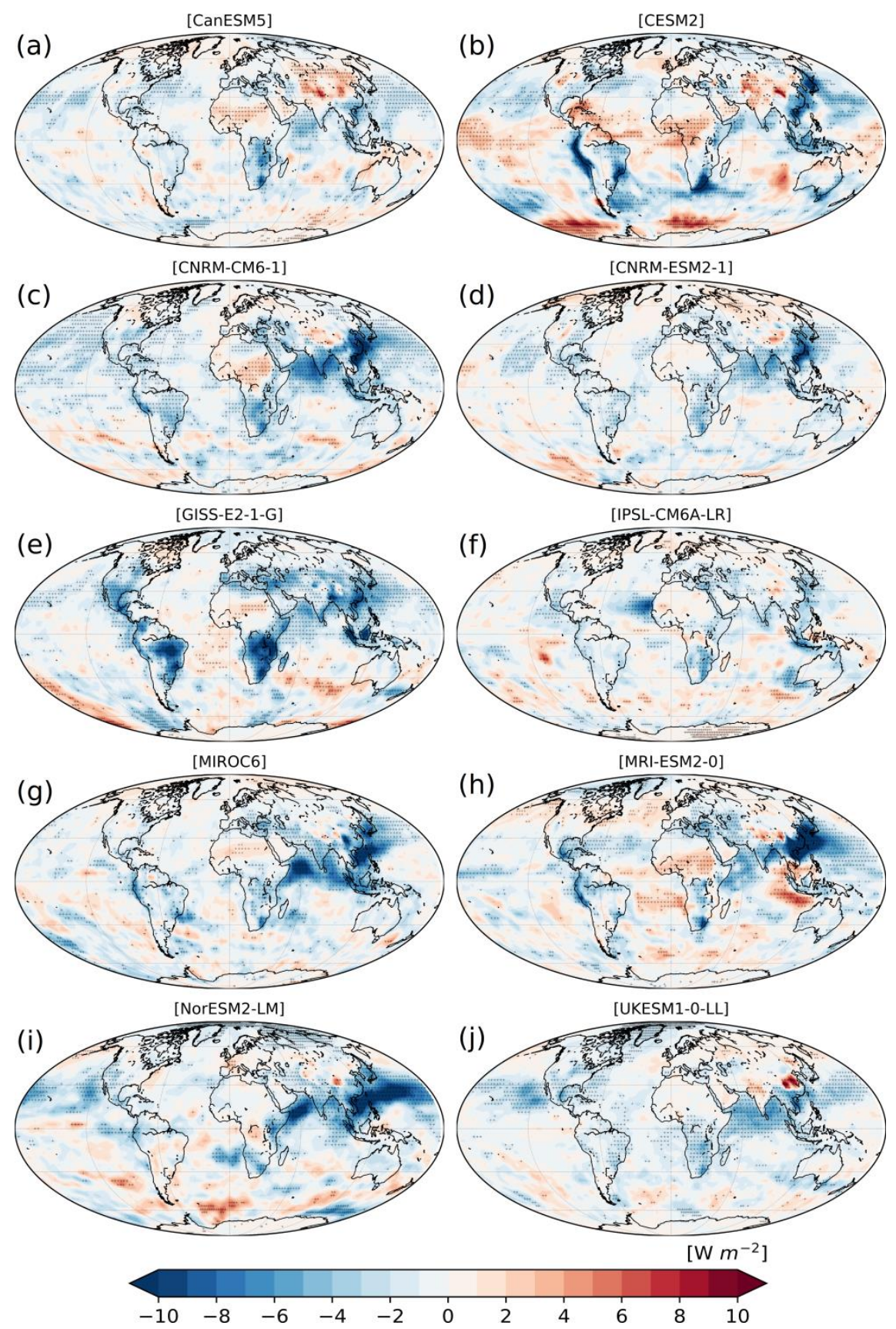

Figure S1. DJF differences between piClim-aer and piClim-control in the net radiative flux (W $\mathrm{m}^{-2}$ ) at TOA including both SW and LW (aerosol ERF) for each one of the models used for the ensemble. The dot shading indicates areas in which the differences are statistically significant at the $95 \%$ confidence level. 
ERF JJA [piClim_aer - piClim_control]
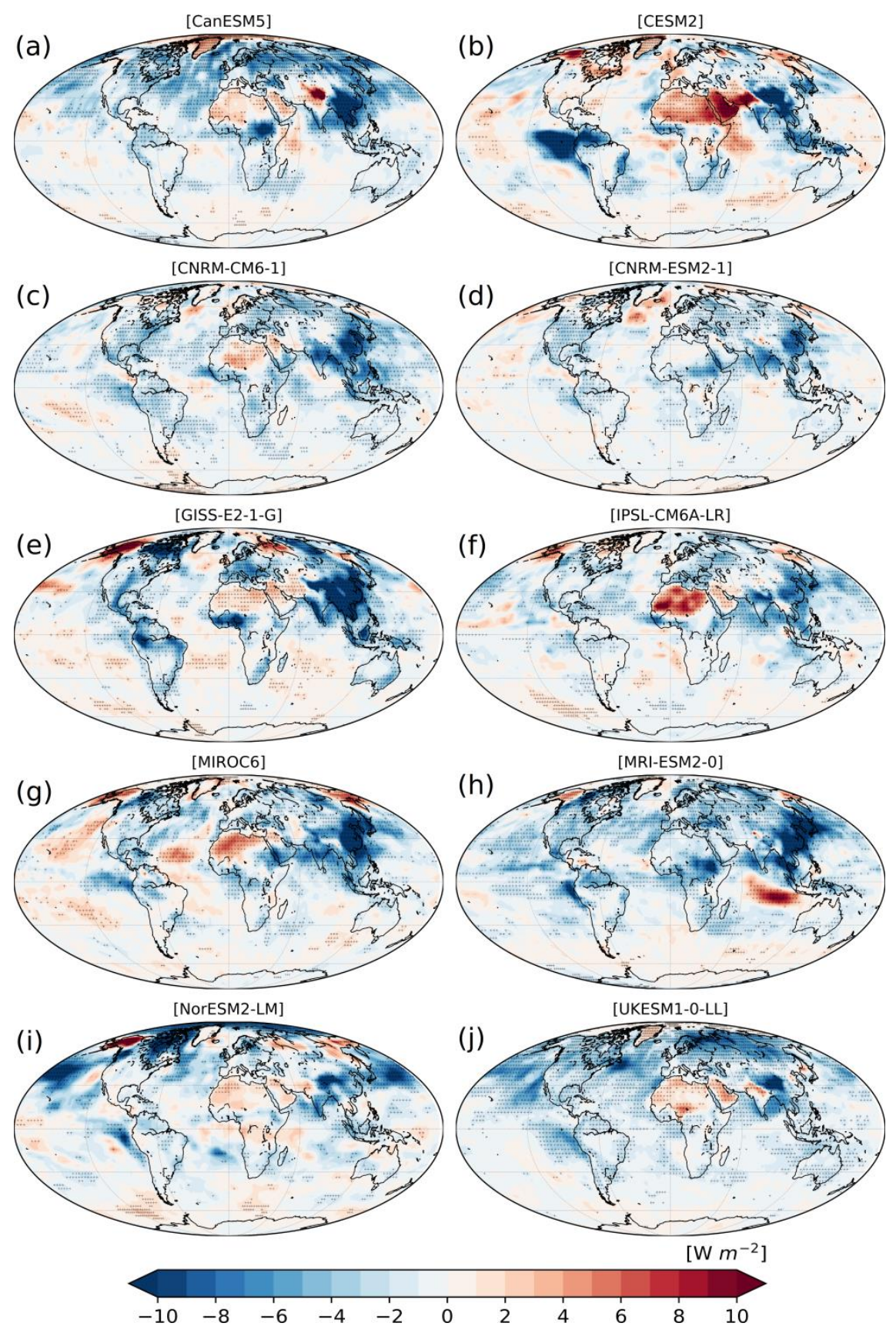

Figure S2. JJA differences between piClim-aer and piClim-control in the net radiative flux (W $\mathrm{m}^{-2}$ ) at TOA including both SW and LW (aerosol ERF) for each one of the models used for the ensemble. The dot shading indicates areas in which the differences are statistically significant at the $95 \%$ confidence level. 


\section{ERF ANNUAL}

[piClim_SO2 - piClim_control] [piClim_BC - piClim_control] [piClim_OC - piClim_control]

(a)

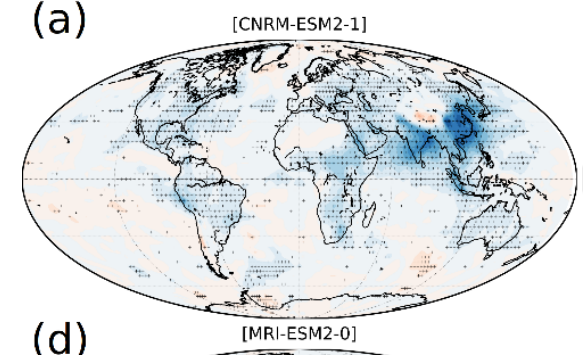

(d)

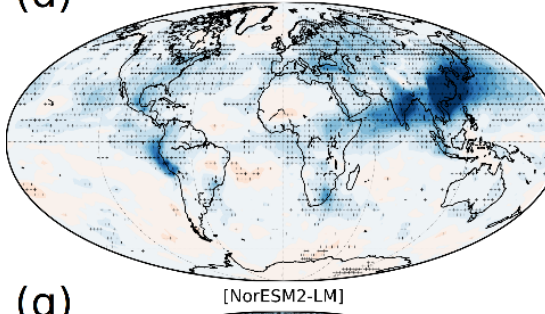

(g)

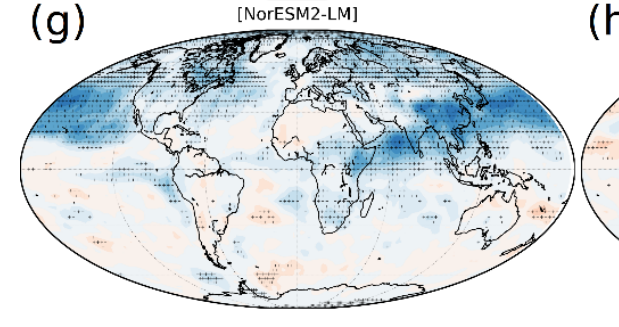

(b)

[CNRM-ESM2-1]

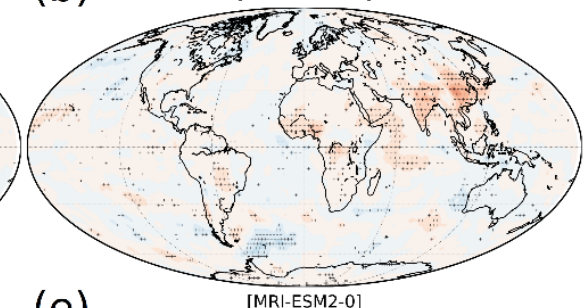

(e)

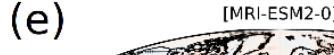

(h) $\frac{1}{\text { [NorESML-LM] }}$

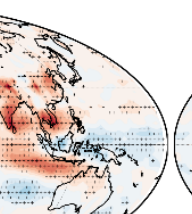

(c) [CNRM-ESM2-1]

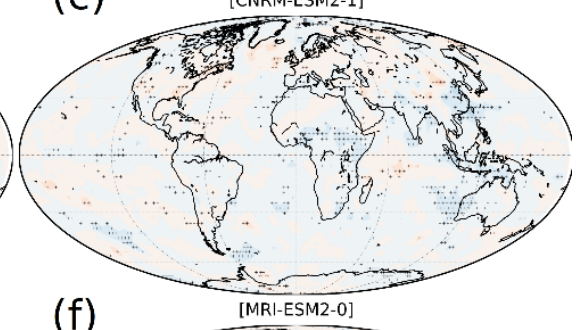

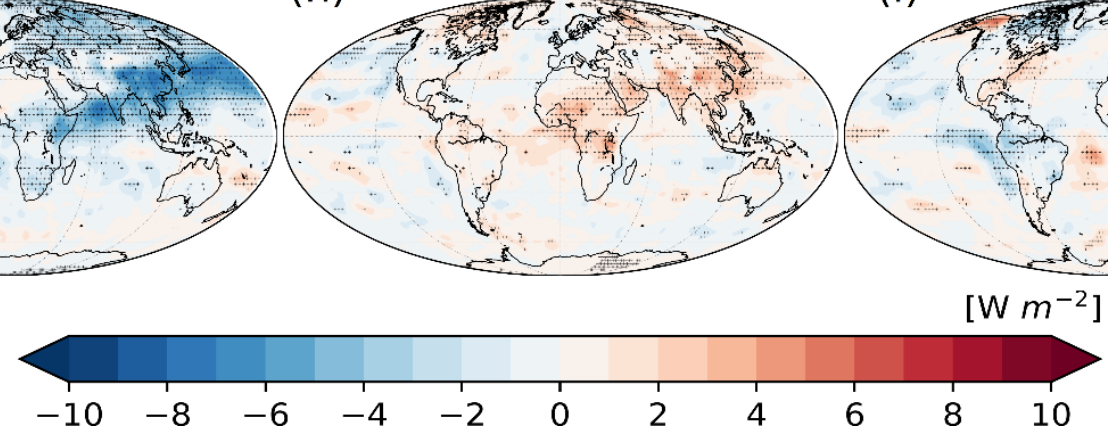

(
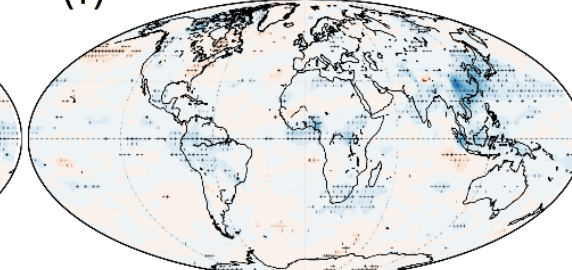

(i)
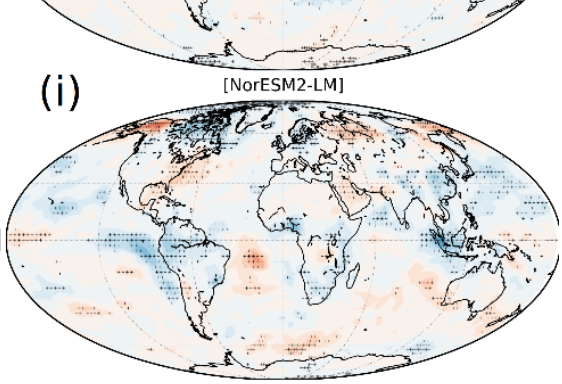

Figure S3. Annual differences in the net radiative flux $\left(\mathrm{W} \mathrm{m}^{-2}\right)$ at TOA including both $\mathrm{SW}$ and LW (ERF) between i) piClim-SO $\mathrm{SO}_{2}$ and piClim-control (first column), ii) piClim-BC and piClimcontrol (second column) and iii) piClim-OC and piClim-control (thrird column) for CNRMESM2-1, MRI-ESM2-0 and NorESM2-LM. The dot shading indicates areas in which the differences are statistically significant at the 95\% confidence level. 
Temperature DJF [piClim_aer - piClim_control]
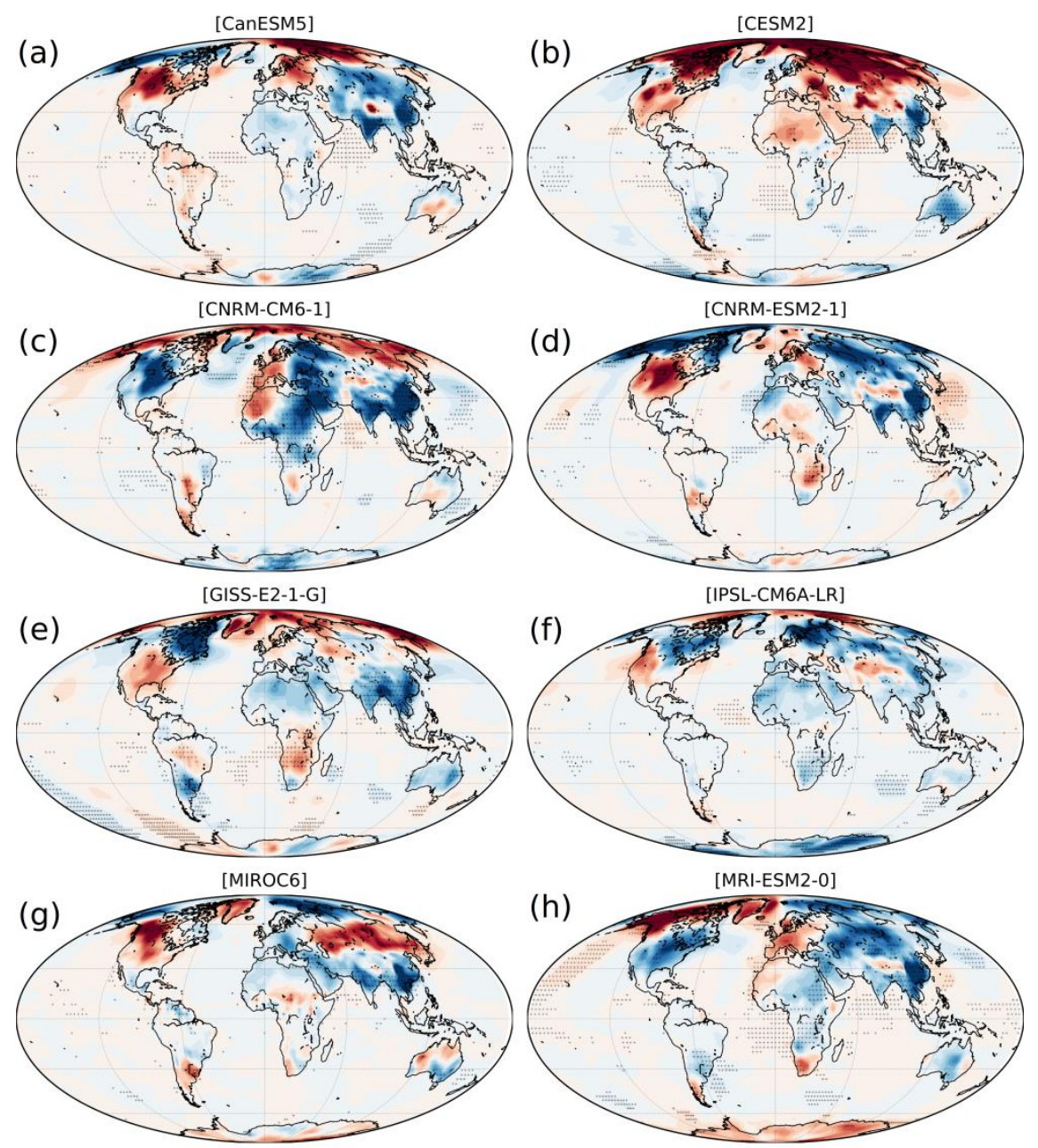

(h)

[MRI-ESM2-0]
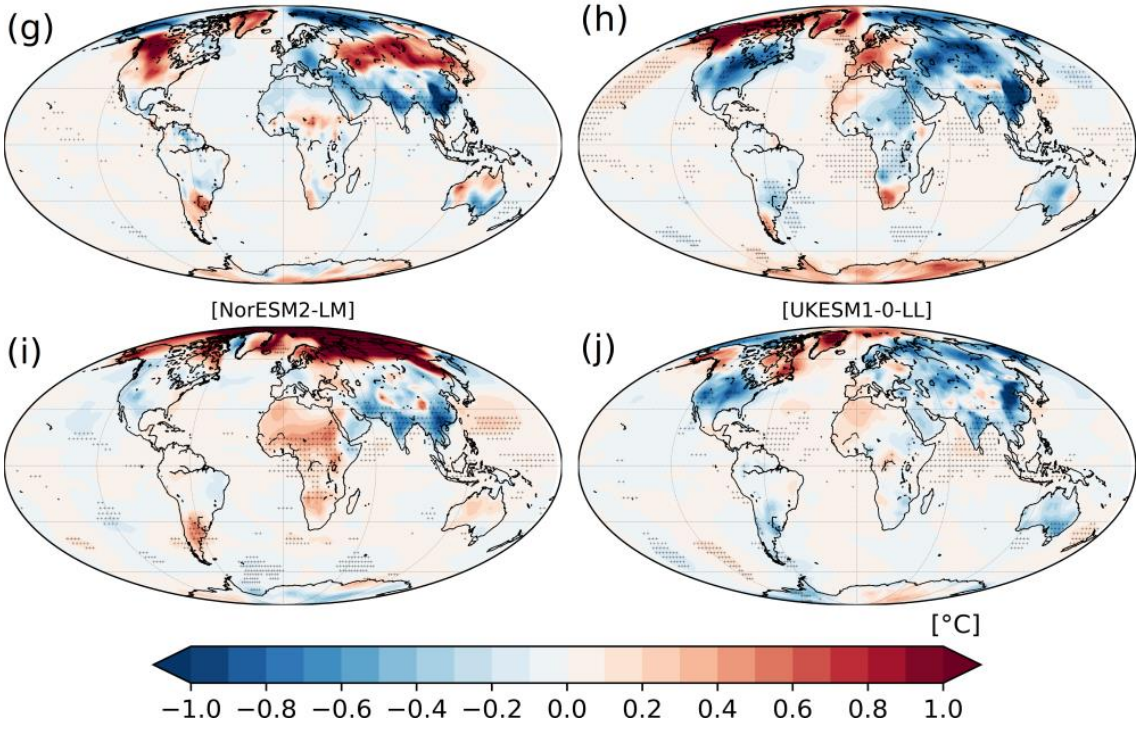

Figure S4. DJF differences between piClim-aer and piClim-control in near surface temperature $\left({ }^{\circ} \mathrm{C}\right)$ for each one of the models used for the ensemble. The dot shading indicates areas in which the differences are statistically significant at the 95\% confidence level. 
Temperature JJA [piClim_aer - piClim_control]
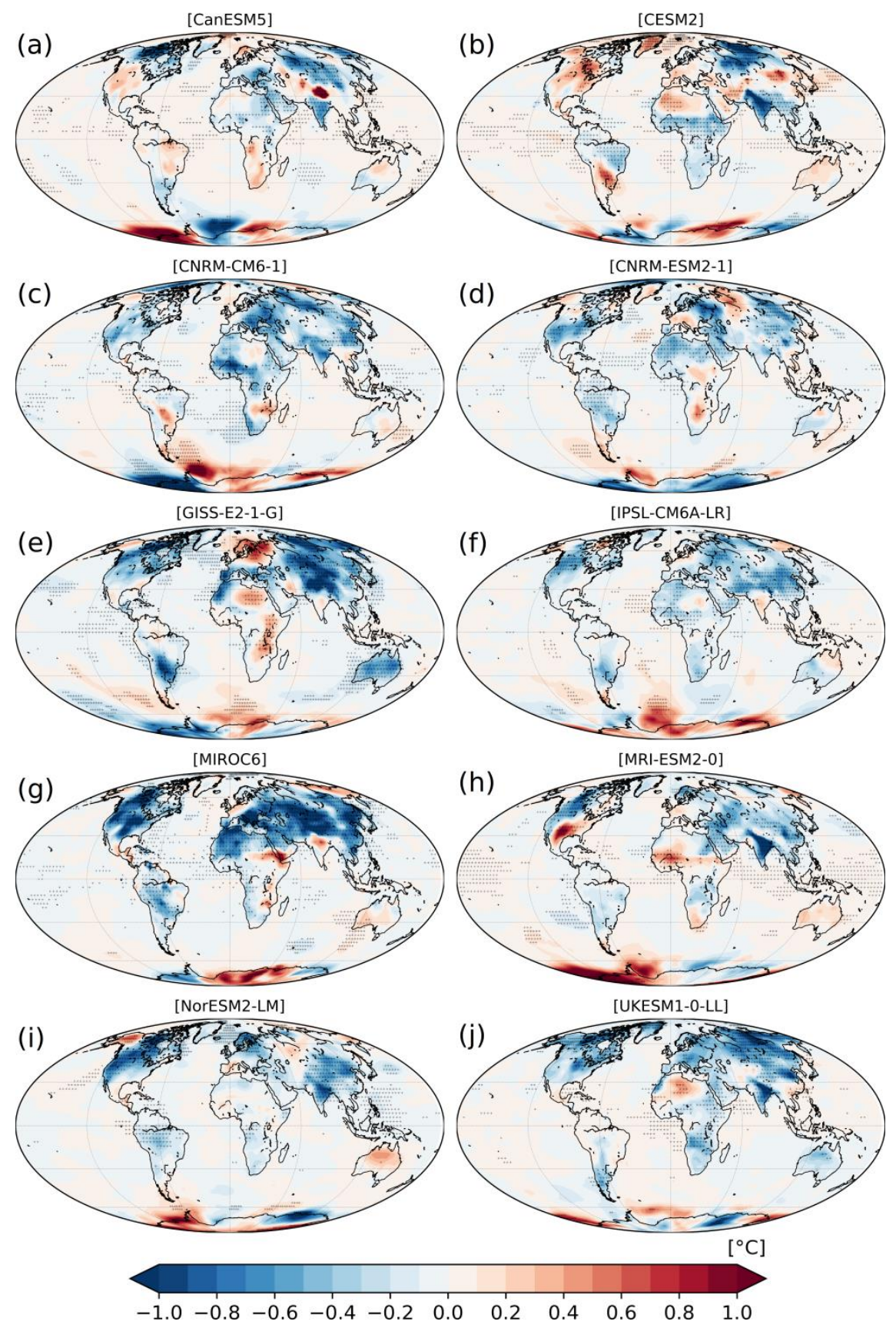

Figure S5. JJA differences between piClim-aer and piClim-control in near surface temperature $\left({ }^{\circ} \mathrm{C}\right)$ for each one of the models used for the ensemble. The dot shading indicates areas in which the differences are statistically significant at the $95 \%$ confidence level. 
Temperature ANNUAL

[piClim_SO2 - piClim_control] [piClim_BC - piClim_control] [piClim_OC - piClim_control]

(a)

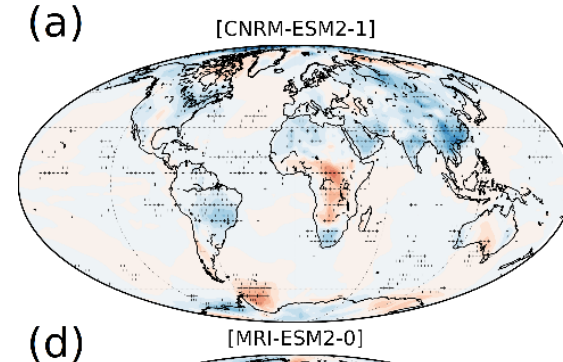

(d)

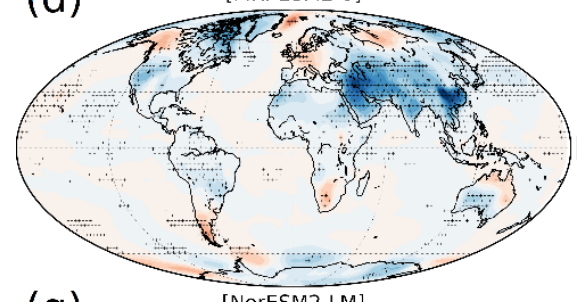

(g)

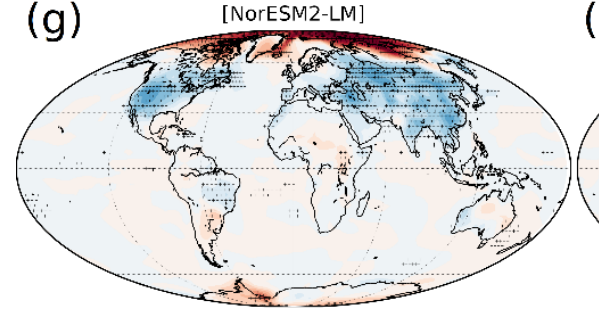

(b)

[CNRM-ESM2-1]

(c)

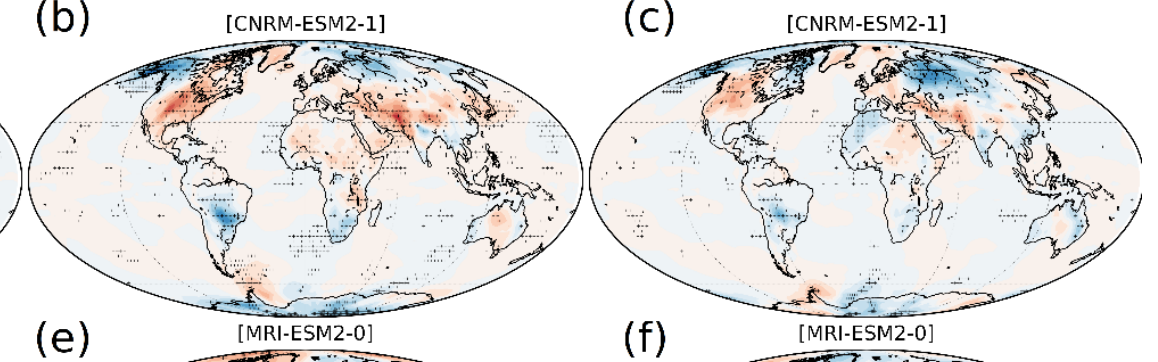

(e) $\frac{1}{\text { [MRI-ESM2-0] }}$

(f)

(h)

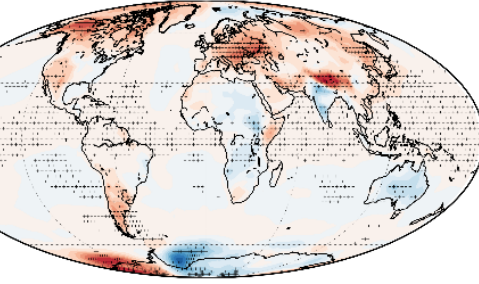

h) [NorESM2-LM]

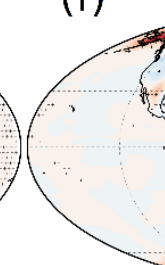

त

(i)

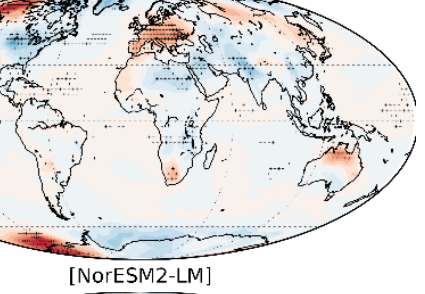

[NorEM LLM]

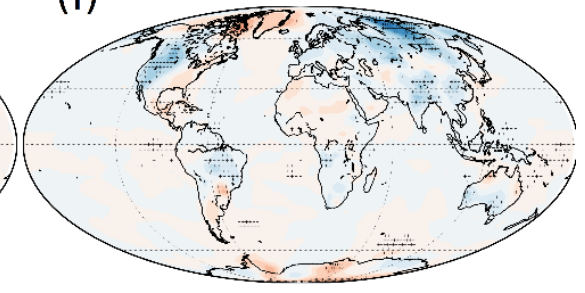

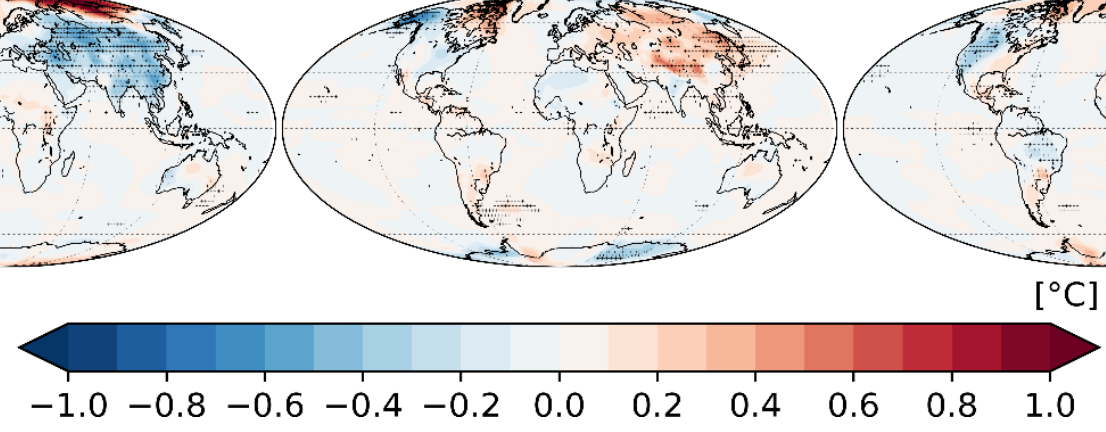

$\left[{ }^{\circ} \mathrm{C}\right]$

Figure S6. Annual differences in near surface temperature $\left({ }^{\circ} \mathrm{C}\right)$ between i) piClim-SO $\mathrm{SO}_{2}$ and piClim-control (first column), ii) piClim-BC and piClim-control (second column) and iii) piClim-OC and piClim-control (thrird column) for CNRM-ESM2-1, MRI-ESM2-0 and NorESM2LM. The dot shading indicates areas in which the differences are statistically significant at the 95\% confidence level. 
Precipitation DJF [piClim_aer - piClim_control]
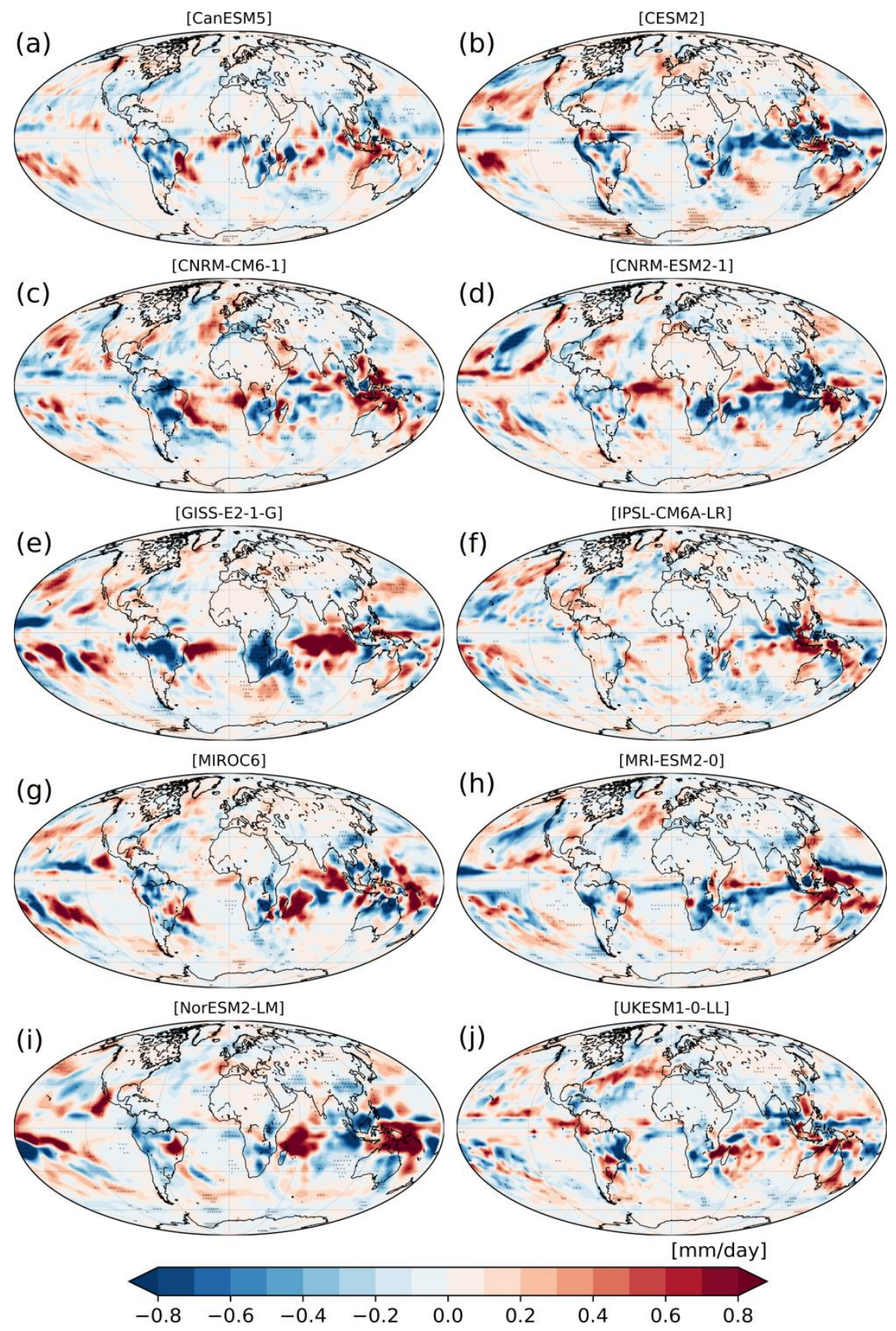

Figure S7. DJF differences between piClim-aer and piClim-control in precipitation (mm/day) for each one of the models used for the ensemble. The dot shading indicates areas in which the differences are statistically significant at the 95\% confidence level. 
Precipitation JJA [piClim_aer - piClim_control]
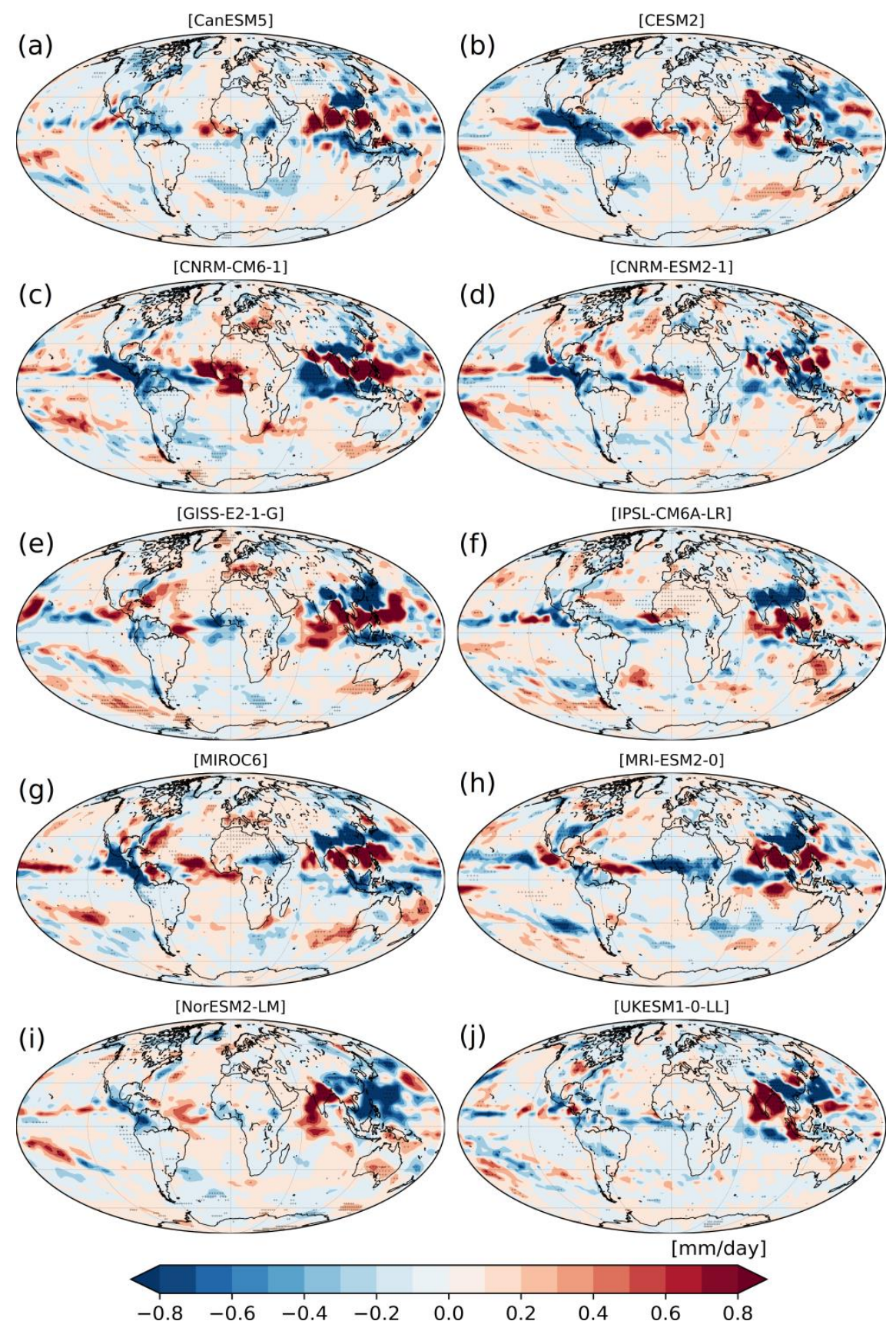

Figure S8. JJA differences between piClim-aer and piClim-control in precipitation (mm/day) for each one of the models used for the ensemble. The dot shading indicates areas in which the differences are statistically significant at the 95\% confidence level. 
Geopot. Height \& Wind DJF [piClim_aer - piClim_control]
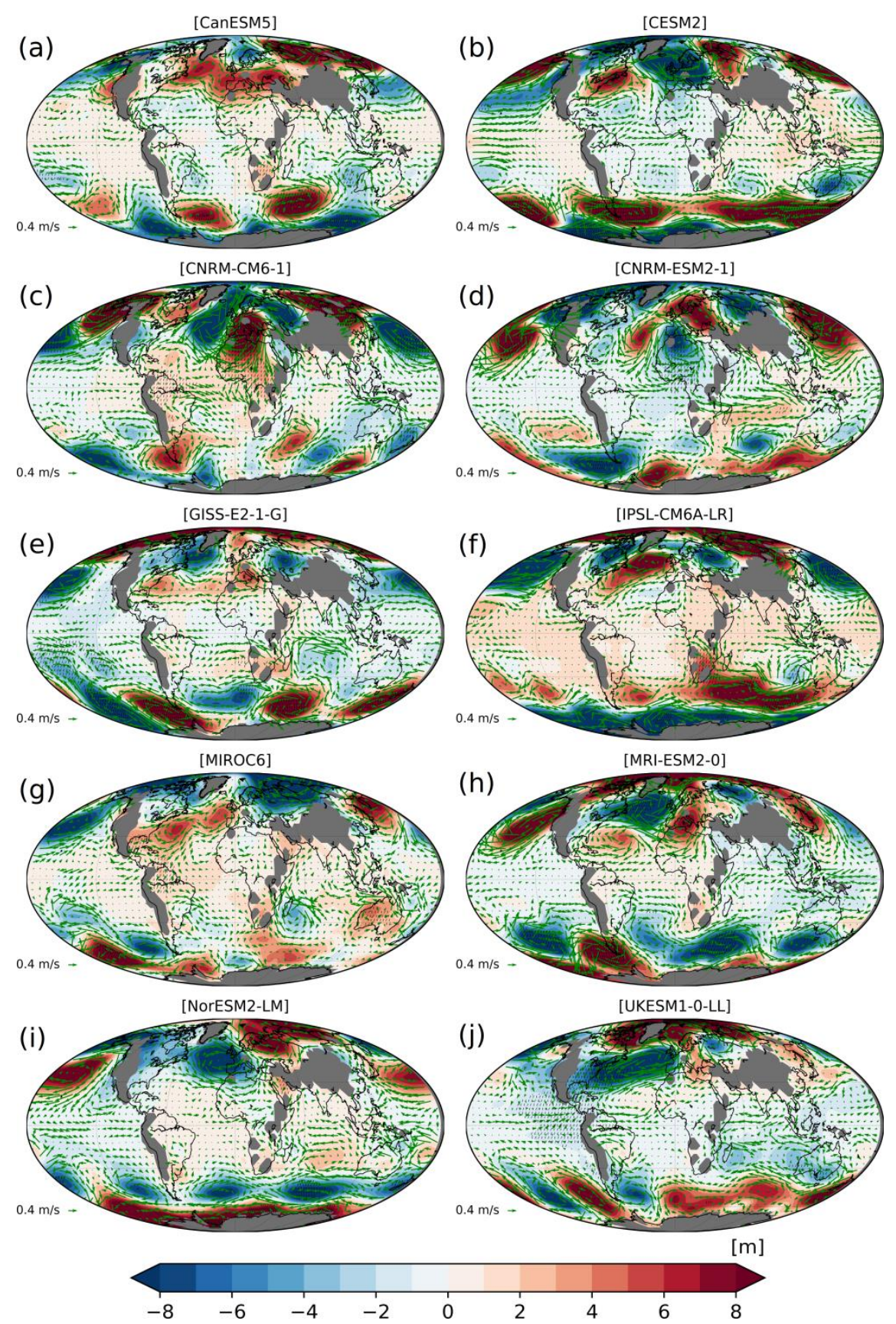

Figure S9. DJF differences between piClim-aer and piClim-control in geopotential height (m) and wind vectors at the $850 \mathrm{hPa}$ pressure level for each one of the models used for the ensemble. The dot shading indicates areas in which the differences are statistically significant at the $95 \%$ confidence level. Areas with surface pressure lower than $850 \mathrm{hPa}$ are masked with grey shade. 
Geopot. Height \& Wind JJA [piClim_aer - piClim_control]
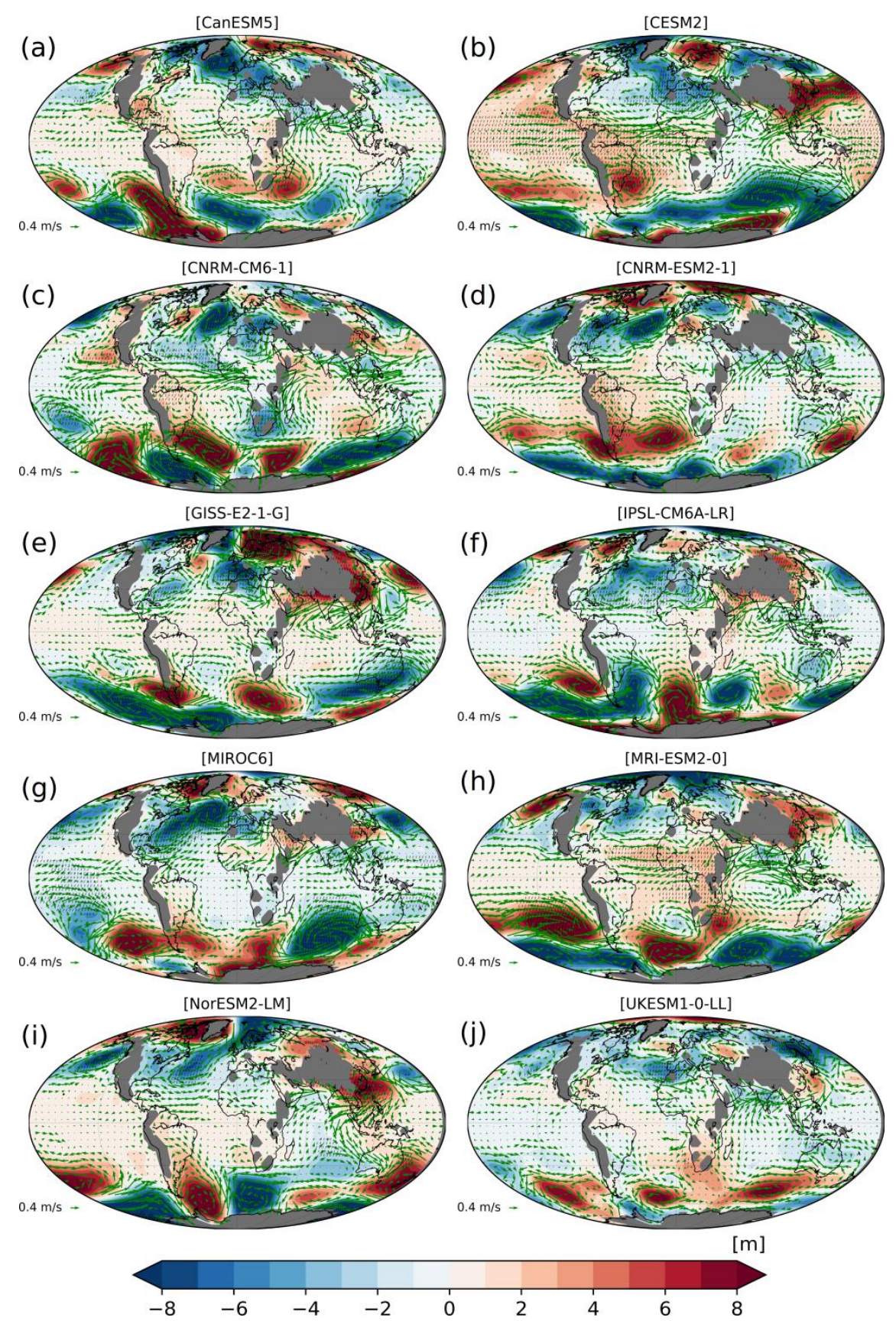

Figure S10. JJA differences between piClim-aer and piClim-control in geopotential height (m) and wind vectors at the $850 \mathrm{hPa}$ pressure level for each one of the models used for the ensemble. The dot shading indicates areas in which the differences are statistically significant at the $95 \%$ confidence level. Areas with surface pressure lower than $850 \mathrm{hPa}$ are masked with grey shade. 